\title{
Reading an augmented reality book: An exploration of learners' cognitive load, motivation, and attitudes
}

\author{
Kun-Hung Cheng \\ National Chiao Tung University
}

\begin{abstract}
Since augmented reality (AR) has been increasingly applied in education recently, the investigation of students' learning experiences with AR could be helpful for educators to implement AR learning. With a quantitative survey using three questionnaires, this study explored the relationships among 153 students' perceived cognitive load, motivation, and attitudes of perceived control, perceived usefulness, behaviour of learning, and behaviour of AR learning, when they engaged in an AR book reading activity. The results indicated that, in general, the students perceived less cognitive load, stronger motivation, and more positive attitudes towards the experiences when reading an AR book. However, dissimilar to past studies, the perceptions of low level cognitive load cannot be guaranteed to lead to positive intentions to learn. Interestingly, motivation mediated the relationships between the students' perceived cognitive load and behavioural intention to learn. Only when the students' perceptions of motivational factors such as attention or confidence were significant did the perceived usefulness and cognitive load play a role in their behavioural intentions to take part in future AR learning. The findings of this study may provide insights for future AR-related studies to explore the role of cognitive load in learning performance with consideration of motivational factors.
\end{abstract}

\section{Introduction}

\section{Augmented reality learning}

According to the Horizon Report by the New Media Consortium (NMC), augmented reality (AR) represents a large amount of the investment undertaken by the technology industry and is indicated as one of the important developments taking place over the next few years (Johnson et al., 2016). Following the trend of technology development, several educational studies have endeavored to explore what role AR, a technique of blending virtual information with the physical environment for presentation in real time, plays in learning. The findings of these studies have mostly indicated the advantages of AR technology, and have further addressed the positive influences of AR on learning effectiveness in fields such as science and engineering learning (e.g., Behzadan \& Kamat, 2013; Chiang, Yang, \& Hwang, 2014; Lu \& Liu, 2015; Sommerauer \& Müller, 2014) and medical science (e.g., Lapeer et al., 2014; Ferrer-Torregrosa, Torralba, Jimenez, García, \& Barcia, 2015). Relatively few studies have probed how AR technology integrated with paper books (called AR books) can assist students' reading. In 2011, Abas and Zaman reported that the AR book system with scaffolding models they developed could help students to read a story book, particularly those with low reading ability. Similarly, in Vate-U-Lan's study (2012), it was found that the students' comprehension of the content of the book was enhanced by using AR technology. A recent study also indicated that, in the AR learning context, children's imagination regarding the content of AR books could be extended (Cheng \& Tsai, 2014). Since the role of AR in reading has been highlighted by previous research, it is suggested that more efforts be made in the study of relevant issues.

\section{Cognitive load}

Reading with the aid of AR may combine the advantages of physical books with digital content including intuitive interaction possibilities (Dünser, 2008). The expansion of the interaction space resulting from connecting book content and digital resources is considered to reduce cognitive load when reading (Prieto, Wen, Caballero, \& Dillenbourg, 2014). Specifically, the AR book systems are capable of bridging the gap between physical and virtual objects and further enhancing readers' understandings of the book content especially in terms of abstract concepts which cannot be comprehended well. For example, students' cognitive overload regarding engineering graphics can be decreased by manipulating and inspecting 3D virtual objects from perspective aspects over the three-view diagrams on a paper book (Martín-Gutiérrez et 
al., 2010). Therefore, learning by AR technology may be helpful for reducing cognitive load and further fostering readers' abilities of understanding abstract concepts or knowledge. Furthermore, the lessening of cognitive load in the limited working memory is supposed to promote the learners' operation of cognitive processes, for example storing knowledge in long-term memory (Santos et al., 2014).

With regard to cognitive load theory, scholars have identified three types of load: intrinsic, extraneous, and germane cognitive load (Sweller, van Merrienboer, \& Paas, 1998). Whereas intrinsic cognitive load is attributed to the difficulty of the learning materials, extraneous cognitive load is attributed to the inappropriate presentation of the instructional materials. Extraneous cognitive load may be caused by cognitive activities that are not directly related to the learning goals. The amount of invested mental effort by learners is considered as germane cognitive load (Plass, Moreno, \& Brünken, 2010). With the capabilities of presenting solid modality and delivering spatial information related to the physical world, it has been asserted that the operation of AR is different from traditional computer interfaces (e.g., keyboard and mouse) and has the potential to enhance learning materials, reduce extraneous cognitive load, and facilitate intrinsic and germane cognitive loads (Bujak et al., 2013; Slijepcevic, 2013). The findings of some empirical studies also correspond to assertions indicating a decrease in cognitive load in AR learning environments (e.g., Klatzky, Wu, Shelton, \& Stetten, 2008; Slijepcevic, 2013; Tang, Owen, Biocca, \& Mou, 2003).

Based on the theory of cognitive load (Plass et al., 2010), learner's perceived cognitive load is deemed as an indicator to predict their learning outcomes by considering the capabilities and limitations of human cognitive architecture. That is to say, less cognitive load could lead to higher learning performance. In the AR-related studies, it has, nevertheless, been found that cognitive load is not related to learning achievement (Küçük, Yilmaz, \& Göktaş, 2014) and cannot predict how learners perform (Chen, Wang, \& Chiang, 2009). That is, the inherent characteristics of AR technology which are the natural interactions with humans do not directly lead to successful learning. Although previous studies have indicated decreased cognitive load in the AR learning context, the above findings may imply that learners' perceived cognitive load is not the sole essential factor affecting their learning outcomes. In addition to the benefits of reducing cognitive load by AR, researchers have suggested that AR applications can motivate users to learn (e.g., Chiang et al., 2014; Ferrer-Torregrosa et al., 2015). Accordingly, the current study proposed that motivational factors should be considered with the measurement of cognitive load for understanding its role in AR-related learning.

\section{Motivation}

In research to date regarding students' motivation when engaging in AR learning, it was reported that the novelty provided by AR is attractive (Pribeanu \& Iordache, 2008) and enjoyable (Wojciechowski \& Cellary, 2013). In an engineering graphics subject for higher education, Martín-Gutiérrez and Contero (2011) found that the university students possessed stronger motivation in settings with AR presentation than when using traditional class notes with static images. Some other AR studies have investigated students' motivation based on Keller's ARCS model (Keller, 1987) which includes four factors: attention, relevance, confidence, and satisfaction (Chiang et al., 2014; Di Serio, Ibáñez, \& Kloos, 2013; Lu \& Liu, 2015). The model describes four steps for facilitating and sustaining motivation in the learning process: (1) attention: gaining learners' attention and arousing their interest in the learning materials, (2) relevance: providing relevant information for learners to fit their personal goals, (3) confidence: establishing positive expectations for achieving success among learners, and (4) satisfaction: feeling satisfied with or gaining rewards from learning experiences.

In their study, Di Serio et al. (2013) found that the ARCS motivational factors of attention and satisfaction were better rated in an AR-based learning environment than in a slides-based learning environment for learning visual arts. When the students' motivation for learning in the AR system was independently surveyed, the attention and confidence factors were highly rated. In other words, the three motivational factors of the ARCS model including attention, confidence, and satisfaction rather than the relevance factor can be highlighted in AR-related learning. Moreover, the results in Chiang et al.'s study (2014) presented that, in the attention, confidence, and relevance dimensions of the ARCS model, the students in the mobile 
AR-based learning groups showed stronger motivation than those in mobile traditional inquiry-based learning groups for learning natural science. The two groups of students in their study did not perceive satisfaction differently. Theoretically, each factor of the ARCS model plays a critical role in motivating students throughout the learning process (Keller, 1987). However, according to the above mentioned studies, it may be argued that learners did not significantly perceive all motivational factors of the ARCS model when involved in different instructional design of the AR learning context.

\section{Cognitive load, motivation, and attitudes}

Previous studies have indicated a probable decrease in cognitive load in AR learning environments (e.g., Klatzky et al., 2008; Slijepcevic, 2013). Minimising cognitive load is generally linked to better learning outcomes, however, in the context of AR learning, the level of cognitive load learners perceived was not directly related to their learning performance (e.g., Küçük et al., 2014). In this regard, Schnotz and Kürschner (2007) reconsidered the cognitive load theory and indicated that the use of individuals' available cognitive capacity is constrained under motivational aspects. The optimisation of cognitive load by instructional manipulations has limited effects on learning unless learners are motivated. The above argument may imply the mediated role of motivation in the relationships between learners' cognitive load and their learning. Paas, Tuovinen, Van Merriënboer, and Darabi (2005) also suggested that the motivational effects of instructional conditions on the examination of learners' cognitive load should be determined, particularly in e-learning environments. With regard to the pedagogical application of AR technology, including motivational factors to discuss with cognitive load may be helpful for gaining a broader understanding of their learning experiences in the AR context (Cheng \& Tsai, 2013).

The process of adapting new technologies by learners is mainly related to their attitudes. The studies based on technology acceptance model (TAM) (Davis, 1989) have documented that learners show positive attitudes when they perceive the new technologies will be easy to use and useful (e.g., Chang, Chen, Huang, \& Huang, 2011; Tsai, Tsai, \& Hwang, 2010). Therefore, in addition to exploring learners' cognitive load and motivation, this study considered that exploring their attitudes toward learning by AR technology is necessary. Some studies have investigated what learners' attitudes toward AR learning systems are. For example, Sin and Zaman (2010) evaluated an AR book-based educational tool they developed and addressed the ease of use and learnability of the tool. Compared with the attitudes toward learning in computer-mediated condition, Han, Jo, Hyun, and So (2015) found that children showed higher satisfaction and perceived engagement in AR-based learning context. Notably, in some studies (e.g., Chang et al., 2011; Wojciechowski \& Cellary, 2013), the factor of perceived usefulness was highlighted as playing a significant role in students' learning by AR technology. Chang et al. (2011) further verified perceived usefulness as a significant indicator to predict students' intention to use AR-learning systems. Accordingly, most of the studies reported the learners' positive attitudes with regard to the usability of the AR system (e.g., the issues of ease of use, usefulness, learnability, or satisfaction) and behavioural intention to use it in the future. However, these studies mostly emphasised the learners' willingness to use the developed system. The investigation regarding learners' behavioural intention to explore more information related to the learning topics or willingness to engage in AR-related learning was relatively limited and worthy of further exploration.

Researchers have found that stronger attitudinal and behavioural effects on the use of media and technology are driven by greater motivation (Rubin, 2002). Several studies also provided evidence indicating the significant role of motivation in users' attitudes toward information technology adoption such as Internet use (Vandenbroeck, Verschelden, \& Boonaert, 2008), social networking site use (Wang, Jackson, Wang, \& Gaskin, 2015), or instant communication service use (Lien \& Cao, 2014). To some extent, these findings have identified the correlations between users' motivation and their attitudes toward technology use. Taking this a step further, it might be valuable to explore how students' motivation links to attitudes toward learning by new technology such as AR. In addition to motivation, the variable of cognitive load was investigated in relation to students' attitudes in the context of AR learning. Küçük et al. (2014) reported that the lower cognitive load students perceived, the more positive attitudes they exhibited. Accordingly, it is plausible to hypothesise that, when engaging in AR learning environments, learners' perceived cognitive load and motivation may play a role in their attitudes. This study considers that discussing cognitive load and 
motivational factors with attitudes (i.e., perceived usability, behavioural intentions to learn in more depth, and behavioural intentions to learn with the aid of AR) can be another angle from which to depict users' perceived AR learning experiences.

\section{Research questions}

As mentioned previously, few studies have explored how AR books can help students' reading. This study therefore attempted to understand students' perceived cognitive load, motivation, and attitudes when reading an AR book. Specifically, with respect to reading an AR book, the research questions of this study are described as follows:

1. What are the students' perceived cognitive load, motivation, and attitudes?

2. What are the relationships among the students' perceived cognitive load, motivation, and attitudes?

3. What roles do the students' perceived cognitive load and motivation play in their attitudes?

\section{Method}

\section{The AR book}

The current study adopted an AR book to investigate users' cognitive load, motivation, and attitudes when engaged in the AR learning context. The AR book system, combining a paper book with an AR App, was established for the purposes of introducing Hakka culture in Taiwan. According to the 2014 census results reported by Hakka Affair Council (2014 Hakka Ethnicity Report, n.d.), Hakka are one of the main cultural and linguistics groups of Chinese ethnicity who make up 18\% of Taiwan's population. The content of the paper book is based on a short novel Tobacco Barn which was written by the writer Chung Li-Ho, an expert in Hakka culture. Learners can read the paper book while using the AR App to focus on the images of the book using a mobile device with a camera (e.g., a smart phone or tablet PC). Subsequently, the virtual information for extending the content of the book would be blended over the pages in the form of 3D models with audio narration and guiding videos. Through gestures, the 3D models can be moved, scaled, and rotated in real time. In addition to the reading of the paper book, learners can receive the augmented information to help them understand Hakka culture in more depth. For example, the book describes the story of the main character getting permission to plant tobacco through the drawing of lots. Reading the book with the AR App can additionally provide learners with information regarding the architecture of a tobacco barn (with 3D models) or the process of planting and baking tobacco leaf (with videos). The augmented information was designed to scaffold the book reading but not to represent the content of the book. The usage of the AR book is presented in Figure 1.

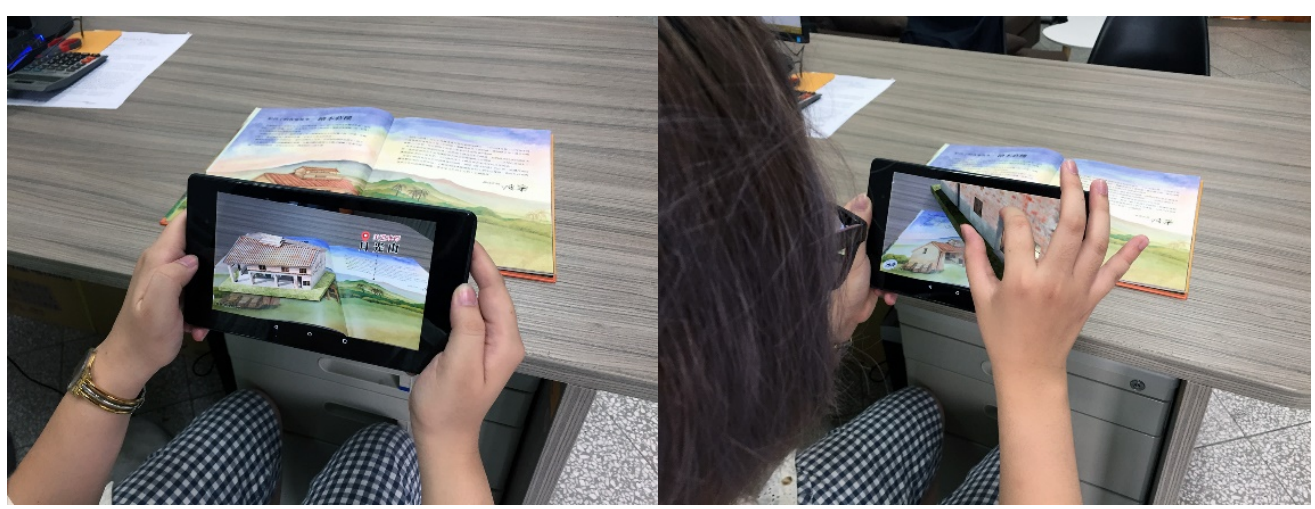

Figure 1. A demonstration of the usage of the AR book.

\section{Sample}

There were 153 university students (including 94 undergraduate students and 59 graduate students) invited to participate in this study. Of these students, 75 were females (49\%) and 78 were males (51\%). Whereas 
68 students were majoring in science and engineering (44\%), 85 were majoring in social science and arts (56\%). Notably, the subject of the AR book is not inherently relevant to the background of the participants in this study. It can be supposed that most of the participants were not familiar with the issues of Hakka culture. To some extent, the participants' prior knowledge about Hakka culture may not play a role in their reading experiences of the AR book. The mean age of these students was close to 22 years old $(S D=2.45)$. According to the demographic data, almost all of the respondents used a smart phone or tablet PC daily (95\%), however, they had relatively less experience of seeing AR (35\%) demonstrations and using AR (30\%) applications. In other words, the students had experience of operating mobile devices but not of using AR technology.

\section{Data collection and instruments}

To collect the data for this study, each respondent was firstly invited to read the AR book using a tablet PC. The reading activity began after a brief introduction of the proper usage of the AR book by a research assistant. When each student had experienced reading the AR book, three instruments for investigating their cognitive load, motivation, and attitudes were administered. The items in the three instruments were presented on a 5-point Likert scale (from 1 - strongly disagree, to 5, strongly agree). Figure 2 illustrates the process of data collection and the instruments used in this study.

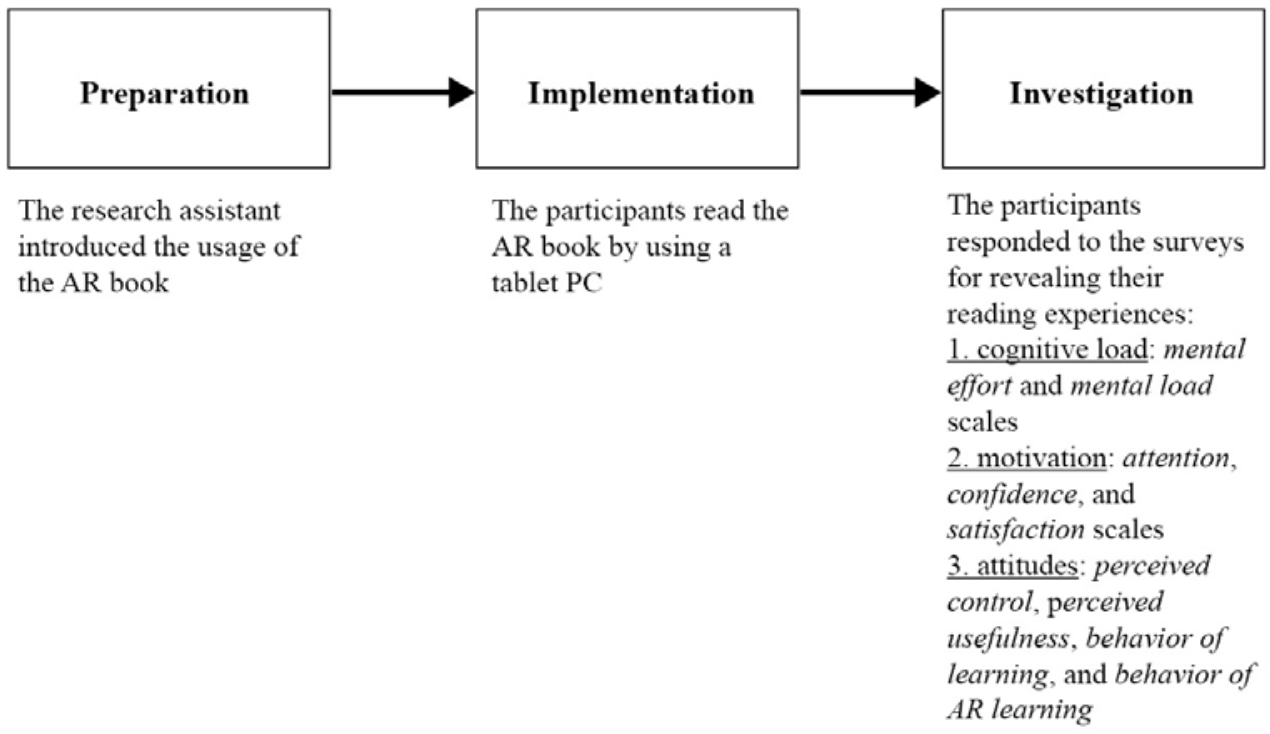

Figure 2. The process of research data collection.

Survey for cognitive load

To understand the students' cognitive load experience, this study adopted the survey used in Hsieh and Tsai's study (2014) and adapted it to the AR book reading context. The cognitive load survey was originally developed by Sweller et al. (1998). There are two scales of mental effort and mental load in the survey. Mental effort measures the extent of an individual's invested cognitive capacity when trying to process information presented in the AR book. Mental load measures the extent of the cognitive capacity which is needed to process the information in the AR book. In this study, high scores for the two scales indicate the students' high cognitive load during the process of reading the AR book. The reliability test showed that the Cronbach's alpha values for the two scales were 0.87 and 0.72 , respectively, and 0.82 for the overall reliability alpha value. The results suggested that the instrument was adequately reliable for investigating the students' cognitive load experience of reading the AR book. Following are sample items of the two scales:

1. Mental effort scale: When I read the AR book, I have to understand the content of the learning material by investing more mental effort.

2. Mental load scale: When I read the AR book, the way in which the learning material is explained gives me a lot of pressure. 


\section{Survey for motivation}

Some AR-related learning studies (Chiang et al., 2014; Di Serio et al., 2013) have measured users' motivation by the instructional materials motivation survey (IMMS) (Keller, 2010) in the light of the ARCS model (Keller, 1987). The IMMS instrument consists of same four scales of motivation: attention, relevance, confidence, and satisfaction. This study previously argued that not all of the motivational factors of the ARCS model would be perceived by learners in different instructional designs of the AR learning context. There were $85 \%$ of the students in this study who considered themselves as not Hakka Taiwanese people, indicating that the students were generally not of Hakka extraction. Moreover, the formal educational programs for introducing Hakka culture were limited in Taiwan. This study therefore supposed that the students were not familiar with Hakka culture (the subject of the AR book); the content of the book was presumed not to relate to the students' lives. As a result, to measure the students' motivation when reading the AR book, this study adopted the three scales, attention, confidence, and satisfaction, but did not use the relevance scale from the IMMS instrument which has been used in Di Serio et al.'s study (2013). Also, the items of the three scales were adapted to the research context of this study. The results of reliability showed that the reliability alpha values for the three scales respectively were $0.82,0.80$, and 0.76 , and the overall reliability alpha value was 0.88 . Therefore, the scales were acceptable for assessing the students' motivation when reading the AR book. The following are sample items:

1. Attention scale: The AR book can keep my attention on the learning materials.

2. Confidence scale: I was confident that the AR technology can help me to read the content of the book.

3. Satisfaction scale: I am satisfied with reading the AR book.

\section{Survey for attitudes}

To assess the students' attitudes toward using mobile devices to read the AR book, the personal digital assistants (PDA) attitude survey generated by Tsai et al. (2010) was adapted to this study. There were four scales in Tsai et al.'s survey (2010): perceived control, perceived usefulness, affection, and behaviour. The affection scale was designed to measure learners' feelings and anxiety when using PDAs (sample item: "In the u-learning environment, I hesitate to use a PDA because of my fear of making mistakes I can't correct”). However, due to the prevalence of mobile devices in recent years, this study considered that the contemporary students' perceptions of anxiety when using mobile devices may not be an urgent issue to explore. Hence, the affection scale in Tsai et al.'s PDA attitude survey (2010) was dismissed from the investigation of this study. The other three scales, perceived control, perceived usefulness, and behaviour, were retained. The behaviour scale in Tsai et al.'s PDA attitude survey (2010) measured the users' degree of willingness to actually use a PDA. In this study, the scale was revised as behaviour of AR learning to measure the students' intention to utilise AR technology to learn in the future. Moreover, to probe the students' behavioural intentions to be involved in a thorough exploration of the issues of Hakka culture after reading the AR book, items were added to construct a new scale named behaviour of learning in this investigation.

Because there were two new scales, behaviour of learning and behaviour of AR learning, established in the survey for the measurement of attitudes, the factor analysis and reliability test were both implemented to confirm the validity and internal consistency of the items. The results of the factor analysis presented that the four scales of the survey: perceived control (4 items), perceived usefulness (4 items), behaviour of learning ( 5 items), and behaviour of AR learning ( 4 items), were respectively identified and showed $64.97 \%$ of the total explained variance. The overall reliability alpha values were $0.87,0.75,0.77,0.89$, and 0.82 , respectively, for each scale, indicating satisfactory internal consistency of the survey. Sample items of the four scales are presented as follows:

1. Perceived control scale: I can learn to use the AR book system in a short time.

2. Perceived usefulness scale: I think the AR book is helpful for my learning.

3. Behaviour of learning scale: I hope to read more information regarding the topic of the AR book.

4. Behaviour of AR learning scale: I hope to have more opportunities to learn with the aid of AR technology. 


\section{Results}

\section{Comparisons of the scales of the three instruments}

To broadly understand the students' cognitive load, motivation, and attitudes when engaging in the AR book reading activity, their scores on the scales of the surveys were examined through the analyses of paired $t$-tests and within-subject ANOVA. According to the data regarding the perceived cognitive load (Table 1), it was shown that the students generally perceived a low level of mental effort $(M=2.23, S D=0.78)$ and mental load $(M=2.20, S D=0.77)$. Moreover, there were no significant differences between the students' perceived mental effort and mental load $(t=0.67, p>0.05)$. That is, when the students read the AR book, they did not perceive cognitive overload when accessing information in the book. The states of the students' invested cognitive efforts (mental effort) and needed cognitive capacity (mental load) during the process of the AR book reading activity were similar.

With regard to the students' attitudes toward reading the AR book, Table 1 presents the differences between the scales in the survey for attitudes. To be more specific, the students' scores on the perceived usefulness scale were higher than their scores on the perceived control scale to a significant level $(t=-3.98, p<0.001)$. That is, the students in this study considered that the book can be more helpful for their learning rather than emphasising the easy control of the AR book. Further examining the students' intention to learn, it was found that their scores on the behaviour of AR learning scale were significantly higher than their scores on the behaviour of learning scale $(t=-3.35, p<0.001$ ). The results indicated that, after reading the AR book, the students were inclined to learn with the aid of AR technology in the future, however, they were relatively less willing to probe more information regarding the topic of the AR book.

Table 1

Paired t-tests for the cognitive load and attitudes surveys

\begin{tabular}{lllr}
\hline Scale & $M$ & $S D$ & $t$ value \\
\hline Cognitive load & & & 0.67 \\
$\quad$ Mental effort & 2.23 & 0.78 & $-3.98^{* * *}$ \\
Mental load & 2.20 & & \\
Attitudes & & & 0.74 \\
Perceived control & 3.73 & 0.53 & $-3.35^{* * *}$ \\
Perceived usefulness & 3.98 & 0.67 & 0.60 \\
Behaviour of learning & 3.60 & & \\
Behaviour of AR learning & 3.79 & & \\
\hline
\end{tabular}

$* * * p<.001$

The comparisons of the scales of the survey for motivation are revealed in Table 2. First of all, the descriptive statistical data reveal that, by and large, the students responded with strong motivation in the dimensions of attention $(M=4.01, S D=0.62)$, confidence $(M=3.81, S D=0.68)$, and satisfaction $(M=$ $3.74, S D=0.68)$. Through the ANOVA analysis, the results show that there were significant differences in the students' responses on the three motivation scales $(F=17.21, p<0.001)$. A series of post hoc tests using Scheffé's method further indicated that the students' attention to the AR book was stronger than their confidence in the advantages of the AR book and their satisfaction with the AR book reading experiences. These findings may imply the role of attention in learners' motivation in the context of AR-related learning. 
Table 2

ANOVA analysis for the motivation survey

\begin{tabular}{lcccc}
\hline Scale & $M$ & $S D$ & $F$ & Post-hoc (Scheffé) \\
\hline Motivation & & & & \\
(1) Attention & 4.01 & 0.62 & $17.21^{* * *}$ & $1>2$ \\
(2) Confidence & 3.81 & 0.68 & & $1>3$ \\
(3) Satisfaction & 3.74 & 0.68 & & \\
\hline
\end{tabular}

$* * * p<.001$

\section{The relationships among the students' cognitive load, motivation, and attitudes}

To examine the relationships among the students' cognitive load, motivation, and attitudes when reading the AR book, a series of Pearson's correlation analyses were conducted and the results are shown in Table 3. It was found that the students' motivation had significant positive relationships with their attitudes. For example, there were moderate correlations between the students' behavioural intention to engage in AR learning in the future and their motivational factors such as attention $(r=0.59, p<0.01)$, confidence $(r=$ $0.63, p<0.01)$, and satisfaction $(r=0.55, p<0.01)$. The students' perceived usefulness also moderately correlated to their attention $(r=0.50, p<0.01)$, confidence $(r=0.50, p<0.01)$, and satisfaction $(r=0.55$, $p<0.01)$. That is, the students with stronger motivation likely perceived the AR book as being more useful for them in consideration of their perceptions of the system control. Moreover, these students also had higher intentions to learn with the aid of $\mathrm{AR}$ in consideration of their willingness to explore more information about Hakka culture.

Table 3 shows that the students' scores on the cognitive load scales were negatively related to their scores on the motivation and attitude scales. In particular, compared with the students' perceived mental load, their mental effort had relatively high negative correlations with their motivation and attitudes. For example, the students' perceptions of mental effort were significantly (negatively) related to their attitudes toward perceived control ( $r=-0.46, p<0.01)$, perceived usefulness $(r=-0.41, p<0.01)$, behaviour of learning $(r$ $=-0.26, p<0.01)$, and behaviour of AR learning $(r=-0.29, p<0.01)$. The findings may indicate that the students with less invested cognitive efforts were inclined to have stronger motivation and more positive attitudes when engaged in the AR book reading activity.

Table 3

The relationships among the students' cognitive load, motivation, and attitudes

\begin{tabular}{|c|c|c|c|c|c|c|c|c|c|}
\hline & PC & PU & BL & BAR & AT & $\mathrm{CO}$ & SA & $\mathrm{ME}$ & ML \\
\hline PC & 1 & & & & & & & & \\
\hline PU & $0.31 * *$ & 1 & & & & & & & \\
\hline BL & $0.22^{* *}$ & $0.51^{* *}$ & 1 & & & & & & \\
\hline BAR & $0.28 * *$ & $0.49 * *$ & $0.40 * *$ & 1 & & & & & \\
\hline AT & $0.20 *$ & $0.50 * *$ & $0.34 * *$ & $0.59 * *$ & 1 & & & & \\
\hline $\mathrm{CO}$ & $0.29 * *$ & $0.50 * *$ & $0.40 * *$ & $0.63 * *$ & $0.60 * *$ & 1 & & & \\
\hline SA & $0.50 * *$ & $0.55^{* *}$ & $0.57 * *$ & $0.55^{* *}$ & $0.55^{* *}$ & $0.58 * *$ & 1 & & \\
\hline $\mathrm{ME}$ & $-0.46 * *$ & $-0.41 * *$ & $-0.26 * *$ & $-0.29 * *$ & $-0.36^{* *}$ & $-0.40 * *$ & $-0.40 * *$ & 1 & \\
\hline ML & $-0.28 * *$ & $-0.30 * *$ & $-0.19 *$ & $-0.25^{* *}$ & $-0.26^{* *}$ & $-0.39 * *$ & $-0.42 * *$ & $0.57 * *$ & 1 \\
\hline
\end{tabular}

Note. PC: perceived control, PU: perceived usefulness, BL: behaviour of learning, BAR: behaviour of AR learning, AT: attention, CO: confidence, SA: satisfaction, ME: mental effort, ML: mental load.

${ }^{* *} p<.01,{ }^{*} p<.05$ 


\section{Path analysis}

Since significant relationships among the students' perceived cognitive load, motivation, and attitudes were found, following the research questions, it is of interest what roles the students' perceived cognitive load and motivation play in their attitudes. As a result, this study conducted a path analysis to examine the structural relationships among these variables. Moreover, the mediating effects of the students' motivation between their cognitive load and attitudes were also explored.

Based on the theory of the TAM, users' behavioural intention to use an information system can be predicted by their perceived ease of use and perceived usefulness (Davis, 1989). The results have been verified by numerous studies in relevant fields (e.g., Park, 2009). Similarly, in this study, the students' attitudes regarding perceived control and perceived usefulness were deemed as independent variables in the path analysis. Moreover, for the measurement of cognitive load, Sweller, Ayres, and Kalyuga (2011) posited that mental effort and mental load are two different but positively correlated constructs. However, some scholars have raised the critical point that the measurement items of cognitive load likely reflect a single construct (Schnotz \& Kürschner, 2007). Respondents may not clearly understand the distinction between the different aspects of cognitive load. Following this assertion, some other studies also combined the two scales of cognitive load as an indicator for data analysis in a virtual reality learning environment (Schrader \& Bastiaens, 2012). Similar to the research context in Schrader and Bastiaens' (2012) study, in the current phase of analysis, the mental effort and mental load scales were therefore merged into a construct of cognitive load.

As shown in Figure 3, the students' behavioural intention to read more information regarding the topics of the AR book was directly predicted by the perception of usefulness $(\beta=0.31, p<0.001)$. When adding the motivational variables as mediators for the analysis, it was found that only the satisfaction factor mediated the relationships between the perceived control ( $\beta=0.30, p<0.001$ ), perceived usefulness $(\beta=0.38, p<$ $0.001)$, cognitive load $(\beta=-0.18, p<0.05)$ and the behavioural intention to learn $(\beta=0.48, p<0.001)$. The results indicate that, firstly, the perceptions regarding how the AR book is helpful for the students can be considered as an important indicator to predict their consequent behaviours to learn about Hakka culture. Secondly, those students perceiving ease of control of the AR book and possessing low level cognitive load may not show a tendency to explore more information regarding Hakka culture unless they were satisfied with or gained some sense of reward from the AR learning experience.

With regard to the students' behavioural intention to learn with the aid of AR, Figure 3 presents that neither perceived usability (i.e., control and usefulness) nor cognitive load were the predictors. However, after taking the mediational effects of motivation into account, it was found that the motivational factor of attention mediated the relationship between the perceived usefulness $(\beta=0.44, p<0.001)$, cognitive load ( $\beta=-0.18, p<0.05$ ), and behavioural intention to engage in AR learning $(\beta=0.26, p<0.01)$. Also, the relationships between the perceived usefulness $(\beta=0.37, p<0.001)$, cognitive load $(\beta=-0.27, p<0.01)$, and behavioural intention to engage in AR learning $(\beta=0.36, p<0.001)$ were mediated by the motivational factor of confidence. That is, the perceptions of usefulness and low cognitive load cannot directly predict the students' expectations to have more opportunities to take part in AR learning. Only when the students' attention can be kept on the learning materials of the AR book, or when their confidence is being benefited by reading the AR book can be guaranteed, could perceived usefulness and cognitive load play a role in the behavioural intentions to adopt AR learning in the future. 


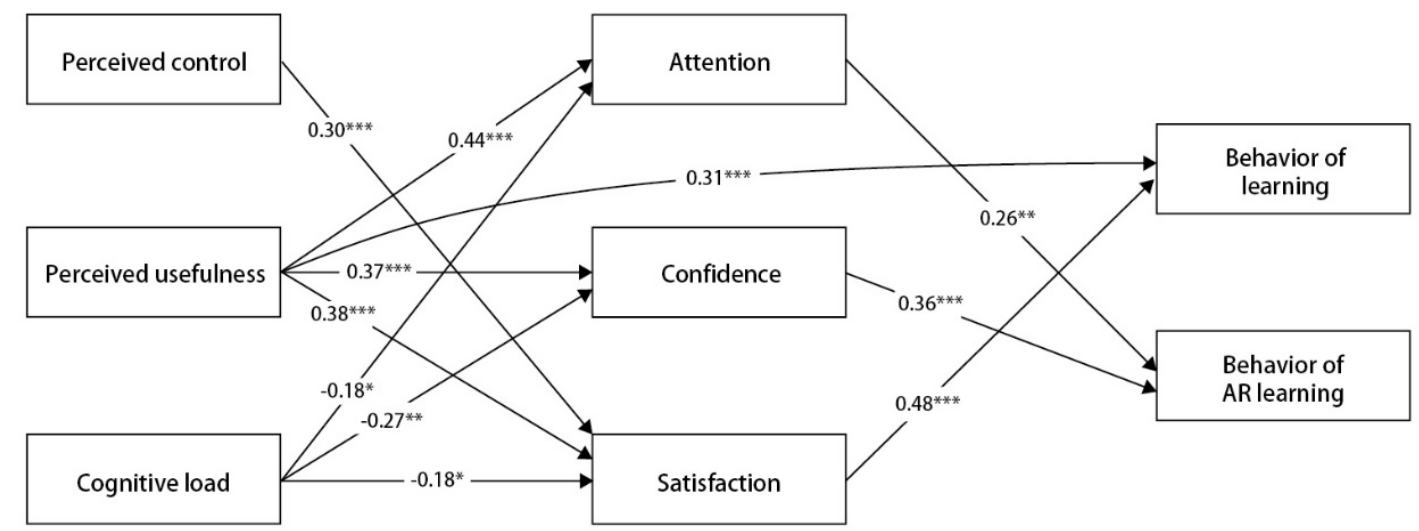

Figure 3. The path analysis for the structural relationships among cognitive load, motivation, and attitudes. ${ }^{* * *} p<.001,{ }^{* *} p<.01,{ }^{*} p<.05$

\section{Discussion and implications}

Based on the previous data analysis, the students' perceived cognitive load, motivation, and attitudes when engaging in the AR book reading were revealed in this study. As for cognitive load, no significant differences between the students' perceived mental effort and mental load were found, and the students' perceived cognitive load in the AR learning activity was generally at a low level. That is, the students did not either need (mental load) or invest (mental effort) much cognitive capacity in processing the information in the AR book. This result corresponds with past studies which reported a decrease in cognitive load in AR learning environments (Klatzky et al., 2008; Tang et al., 2003). In this study, the students were not familiar with the Hakka cultural issues presented by the AR book. It was supposed that they may possess intrinsic cognitive load when reading an unfamiliar topic and have extraneous cognitive load which was resulted by novel reading (note that the content of the AR book used in this study was organised in short novel format). Previous studies regarding AR learning have indicated that, dissimilar with electronic book reading, the interaction between virtual information and physical world created by AR books may enrich users' learning experiences (e.g., Cheng \& Tsai, 2014). The interaction generated by AR learning could leverage users' knowledge from the real world (e.g., Hornecker \& Dünser, 2009). Accordingly, with the intervention of AR technology, the previous argument may imply a possibility to reduce extraneous cognitive load when reading paper books (Slijepcevic, 2013).

The students' attitudes toward the AR book reading activity in this study were positive. In particular, they considered the AR book as helpful for their reading. Similarly, in Wojciechowski and Cellary's study (2013), the students' perceived usefulness rather than their perceived control of the AR environment was also highlighted. Moreover, the students had a stronger tendency to accept AR-related learning in the future (behaviour of AR learning) than to explore Hakka culture in more depth (behaviour of learning). These results may imply that the students had more interest in experiencing the possibility of AR technology for enhancing their learning. In other words, the new way of interaction resulting from connecting book content and digital information is likely more attractive for the students. In addition, the students also responded with strong motivation when reading the AR book, especially in the dimension of attention. Compared with the motivational factors of confidence and satisfaction, they strongly considered that the information in the AR book attracted their attention during the reading process. These results were also similar to Di Serio et al.'s findings (2013) and may imply the importance of perceived attention for learning in an AR context.

Furthermore, in this study, the scales of cognitive load, motivation, and attitudes were significantly correlated to each other. To be more specific, the students with less cognitive load may have stronger motivation and more positive attitudes when engaging in the AR book reading activity. However, according to the path analysis, the students' perceived cognitive load cannot directly predict their attitudes regarding behavioural intentions. In other words, although the students read the AR book with less invested mental 
efforts, the perceptions of low level cognitive load cannot be guaranteed to lead to positive intentions to learn in the future. Previous studies regarding AR learning also addressed similar results that cognitive load was not related to learning achievement (Chen et al., 2009; Küçük et al., 2014). Interestingly, in consideration of motivation as the mediator, this study further found that the students' motivational factors may mediate the relationships between their perceived cognitive load and behavioural intentions. That is, the students who not only perceived a low level cognitive load but also had strong motivation would show a tendency to learn more about Hakka culture or to participate in other AR learning activities. These results may provide insights for future AR-related studies to explore the role of cognitive load in learning performance with consideration of motivational factors.

Finally, based on the findings of this study, the following implications are proposed. For educators or instruction designers who intend to create AR learning environments, in addition to taking advantage of an AR interface to reduce the cognitive load, the motivational factors of attention and confidence should also be considered. It is understandable that the augmented objects or elements blending with the physical world are attractive for users in an AR learning context. However, the effects of the novelty of experiencing AR technology are expected to decrease as learners become increasingly familiar with its presentation form. Taking the design of AR books for example, to sustain users' attention and continuingly arouse their interest in the learning materials, this study suggests that appropriate questions or prompts related to the content of the book can be provided with the forms of augmented information. In addition to operating the augmented objects, the learners need to pay attention to reading the paper book and explore relevant information regarding the questions or prompts. Accordingly, the learners have more opportunities to continuously interact with the learning materials in AR books. Besides the promotion of attention, solutions to the questions or guidance via prompts could also be provided to establish the learners' confidence in achieving success.

\section{Conclusion and future work}

The present study looks at the technological area of AR that is currently rushing to develop applications for learning. The relationships among the students' learning experiences including variables of perceived cognitive load, motivation, and attitudes when engaged in an AR book reading activity were accordingly explored. The roles that cognitive load and motivation played in the students' attitudes were also identified. In view of the increasing efforts to explore how AR technology can help learning (e.g., Lu \& Liu, 2015), particularly AR book reading (e.g., Cheng \& Tsai, 2014), the current study could provide more understanding of the students' perceptions of AR book reading. To contribute to the following research on AR learning, the direction of future work is proposed. First, in addition to the variables investigated in this study, to thoroughly understand users' perceptions of AR learning, user experience (UX) factors or conceptions of AR learning could be included for constructing a structural model of AR learning experience. Second, the research method of thinking aloud while reading AR books or interviewing learners after reading activities could be implemented in future work. The qualitative data could reveal the learners' thinking during the AR book reading process. The understanding of learners' perceptions of AR learning would be fulfilled. Third, an objective measurement of cognitive load such as neuro-imaging techniques (Brunken, Plass, \& Leutner, 2003) can be considered in future studies. Through analysing how the brain works during the task, learners' variation of cognitive capacity could be detected and their perceived cognitive load in AR learning environments could accordingly be better understood. Finally, in this study, the topic of the AR book is not inherently relevant to the background of the participants and therefore, the scale of relevance in the motivation measurement was dismissed. However, exploring the students' motivation to AR learning without measuring their perceived degree of how the learning materials were relevant to them might be a limitation of this study. Future work could consider to include the scale of relevance to measure students' motivation. For example, the pre-posttest research design can examine the differences of students' motivation, particularly for the perceptions of how well the content of AR books meets their needs and goals, before and after reading AR books consisting of the content they are unfamiliar with. In addition, a pretest to measure the participants' prior knowledge regarding Hakka culture could be considered in future work. It is also suggested to invite subjects who are familiar with the topic of the AR book (Hakka culture) or who belong to Hakka ethnic group for further exploring their reading experiences. 


\section{Acknowledgments}

This study is supported by the Hakka Affairs Council, Taiwan and in part by Ministry of Science and Technology, Taiwan under grant number MOST 103-2511-S-009-010.

\section{References}

Abas, H., \& Zaman, H. B. (2011). Visual learning through augmented reality storybook for remedial student. Proceedings of the Second International Conference on Visual Informatics: Sustaining Research and Innovations, Selangor, Malaysia, 157-167. https://doi.org/10.1007/978-3-642-25200$\underline{6 \_16}$

Behzadan, A. H., \& Kamat, V. R. (2013). Enabling discovery-based learning in construction using telepresent augmented reality. Automation in Construction, 33, 3-10. https://doi.org/10.1016/j.autcon.2012.09.003

Brunken, R., Plass, J. L., \& Leutner, D. (2003). Direct measurement of cognitive load in multimedia learning. Educational Psychologist, 38(1), 53-61. https://doi.org/10.1207/S15326985EP3801_7

Bujak, K. R., Radu, L., Catrambone, R., MacIntyre, B., Zheng, R., \& Golubski, G. (2013). A psychological perspective on augmented reality in the mathematics classroom. Computers \& Education, 68, 536-544. https://doi.org/10.1016/j.compedu.2013.02.017

Chang, Y. J., Chen, C. H., Huang, W. T., \& Huang, W. S. (2011). Investigating students' perceived satisfaction, behavioral intention, and effectiveness of English learning using augmented reality. Proceedings of the 2011 IEEE International Conference on Multimedia and Expo, Barcelona, Spain, 1-6. https://doi.org/10.1109/ICME.2011.6012177

Chen, Y. C., Wang, S. J., \& Chiang, Y. L. (2009). Exploring the effect of presence in an AR-based learning environment. Proceedings of the 13th Global Chinese Conference on Computers in Education, Taipei, Taiwan, 693-702. http://citeseerx.ist.psu.edu/viewdoc/download?doi=10.1.1.647.633\&rep=rep1\&type=pdf

Cheng, K. H., \& Tsai, C. C. (2013). Affordances of augmented reality in science learning: Suggestions for future research. Journal of Science Education and Technology, 22(4), 449-462. https://doi.org/10.1007/s10956-012-9405-9

Cheng, K. H., \& Tsai, C. C. (2014). Children and parents' reading of an augmented reality picture book: Analyses of behavioral patterns and cognitive attainment. Computers \& Education, 72, 302-312. https://doi.org/10.1016/j.compedu.2013.12.003

Chiang, T. H. C., Yang, S. J. H., \& Hwang, G. J. (2014). An augmented reality-based mobile learning system to improve students' learning achievements and motivations in natural science inquiry activities. Educational Technology \& Society, 17(4), 352-365. Retrieved from http://www.ifets.info/journals/17 4/24.pdf

Davis, F. D. (1989). Perceived usefulness, perceived ease of use, and user acceptance of information technology. MIS Quarterly, 13(3), 319-340. Retrieved from http://www.jstor.org/stable/249008

Di Serio, A., Ibáñez, M. B., \& Kloos, C. D. (2013). Impact of an augmented reality system on students' motivation for a visual art course. Computers \& Education, 68, 586-596. https://doi.org/10.1016/j.compedu.2012.03.002

Dünser, A. (2008). Supporting low ability readers with interactive augmented reality. Annual Review of CyberTherapy and Telemedicine, 6, 41-48. Retrieved from http://www.arctt.info/volume-6--summer2008

Ferrer-Torregrosa, J., Torralba, J., Jimenez, M. A., García, S., \& Barcia, J. M. (2015). ARBOOK: Development and assessment of a tool based on augmented reality for anatomy. Journal of Science Education and Technology, 24(1), 119-124. https://doi.org/10.1007/s10956-014-9526-4

Hakka Affair Council. (n.d.). 2014 Hakka ethnicity census report. Retrieved from http://web3.hakka.gov.tw/ct.asp?xItem=43944\&ctNode=2908\&mp=2013\&ps $=$

Han, J., Jo, M., Hyun, E., \& So, H. J. (2015). Examining young children’s perception toward augmented reality-infused dramatic play. Educational Technology Research and Development, 63(3), 455-474. https://doi.org/10.1007/s11423-015-9374-9

Hornecker, E., \& Dünser, A. (2009). Of pages and paddles: Children's expectations and mistaken interactions with physical-digital tools. Interacting with Computers, 21(1-2), 95-107. 
https://doi.org/10.1016/j.intcom.2008.10.007

Hsieh, Y. H., \& Tsai, C. C. (2014). Students' scientific epistemic beliefs, online evaluative standards, and online searching strategies for science information: The moderating role of cognitive load experience. Journal of Science Education and Technology, 23(3), 299-308. https://doi.org/10.1007/s10956-013$\underline{9464-6}$

Johnson, L., Adams-Becker, S., Cummins, M., Estrada, V., Freeman, A., \& Hall, C. (2016). NMC Horizon Report: 2016 Higher Education Edition. Austin, TX: The New Media Consortium. Retrieved from http://cdn.nmc.org/media/2016-nmc-horizon-report-he-EN.pdf

Keller, J. M. (1987). Development and use of the ARCS model of motivational design. Journal of Instructional Development, 10(3), 2-10. https://doi.org/10.1007/BF02905780

Keller, J. M. (2010). Motivational design for learning and performance: The ARCS model approach. New York, NY: Springer.

Klatzky, R. L., Wu, B., Shelton, D., \& Stetten, G. (2008). Effectiveness of augmented reality visualization versus cognitive mediation for learning actions in near space. ACM Transactions on Applied Perception (TAP), 5(1), 1-23. https://doi.org/10.1145/1279640.1279641

Küçük, S., Yilmaz, R. M., \& Göktaş, Y. (2014). Augmented reality for learning English: Achievement, attitude and cognitive load levels of students. Education and Science, 39(176), 393-404. https://doi.org/10.15390/EB.2014.3595

Lapeer, R. J, Jeffrey, S. J., Dao, J. T., García, G. G., Chen, M., \& Shickell, S. M. (2014). Using a passive coordinate measurement arm for motion tracking of a rigid endoscope for augmented-reality imageguided surgery. The International Journal of Medical Robotics and Computer Assisted Surgery, 10(1), 65-77. https://doi.org/10.1002/rcs.1513

Lien, C. H., \& Cao, Y. (2014). Examining WeChat users' motivations, trust, attitudes, and positive wordof-mouth: Evidence from China. Computers in Human Behavior, 41, 104-111. https://doi.org/10.1016/j.chb.2014.08.013

Lu, S. J., \& Liu, Y. C. (2015). Integrating augmented reality technology to enhance children's learning in marine education. Environmental Education Research, 21(4), 525-541. https://doi.org/10.1080/13504622.2014.911247

Martín-Gutiérrez, J., \& Contero, M. (2011). Improving academic performance and motivation in engineering education with augmented reality. Communications in Computer and Information Science, 174(Part 2), 509-513. https://doi.org/10.1007/978-3-642-22095-1_102

Martín-Gutiérrez, J., Luís Saorín, J., Contero, M., Alcañiz, M., Pérez-López, D. C., \& Ortega, M. (2010). Design and validation of an augmented book for spatial abilities development in engineering students. Computers and Graphics, 34(1), 77-91. https://doi.org/10.1016/j.cag.2009.11.003

Paas, F., Tuovinen, J. E., Van Merriënboer, J. J. G., \& Darabi, D. (2005). A motivational perspective on the relation between mental effort and performance: Optimizing learner involvement in instruction. Educational Technology Research and Development, 53(3), 25-34. https://doi.org/10.1007/BF02504795

Park, S. Y. (2009). An analysis of the technology acceptance model in understanding university students' behavioral intention to use e-learning. Educational Technology \& Society, 12(3), 150-162. Retrieved from http://www.ifets.info/journals/12_3/14.pdf

Plass, J. L., Moreno, R., \& Brünken, R. (2010). Cognitive load theory. New York, NY: Cambridge University Press.

Pribeanu, C., \& Iordache, D. D. (2008). Evaluating the motivational value of an augmented reality system for learning chemistry. Proceedings of the 4th Symposium of the Workgroup Human-Computer Interaction and Usability Engineering of the Austrian Computer Society on HCI and Usability for Education and Work, Graz, Austria, 31-42. https://doi.org/10.1007/978-3-540-89350-9_3

Prieto, L.-P., Wen, Y., Caballero, D., \& Dillenbourg, P. (2014). Review of augmented paper systems in education: An orchestration perspective. Educational Technology \& Society, 17(4), 169-185. Retrieved from http://www.ifets.info/journals/17 4/11.pdf

Rubin, A. M. (2002). The uses-and-gratifications perspective of media effects. In J. Bryant \& D. Zillmann (Eds.), Media effects: Advances in theory and research (2nd ed., pp. 525-548). Hillsdale, NJ: Lawrence Erlbaum Associates Publishers.

Santos, M. E. C., Chen, A., Taketomi, T., Yamamoto, G., Miyazaki, J., \& Kato, H. (2014). Augmented reality learning experiences: Survey of prototype design and evaluation. IEEE Transactions on 
Learning Technologies, 7(1), 38-56. https://doi.org/10.1109/TLT.2013.37

Schnotz, W., \& Kürschner, C. (2007). A reconsideration of cognitive load theory. Educational Psychology Review, 19(4), 469-508. https://doi.org/10.1007/s10648-007-9053-4

Schrader, C., \& Bastiaens, T. J. (2012). The influence of virtual presence: Effects on experienced cognitive load and learning outcomes in educational computer games. Computers in Human Behavior, 28(2), 648-658. https://doi.org/10.1016/j.chb.2011.11.011

Sin, A. K., \& Zaman, H. B. (2010). Live solar system (LSS): Evaluation of an augmented reality bookbased educational tool. Proceedings of the 2010 International Symposium on Information Technology - Visual Informatics, Kuala Lumpur, Malaysia, 1-6. https://doi.org/10.1109/ITSIM.2010.5561320

Slijepcevic, N (2013). The effect of augmented reality treatment on learning, cognitive load, and spatial visualization abilities (Unpublished doctoral dissertation). University of Kentucky, Lexington, KY.

Sommerauer, P., \& Müller, O. (2014). Augmented reality in informal learning environments: A field experiment in a mathematics exhibition. Computers \& Education, 79, 59-68. https://doi.org/10.1016/j.compedu.2014.07.013

Sweller, J., Ayres, P., \& Kalyuga, S. (Eds.). (2011). Cognitive load theory. New York, NY: Springer.

Sweller, J., van Merrienboer, J., \& Paas, F. (1998). Cognitive architecture and instructional design. Educational Psychology Review, 10(3), 251-296. https://doi.org/10.1023/A:1022193728205

Tang, A., Owen, C., Biocca, F., \& Mou, W. (2003). Comparative effectiveness of augmented reality in object assembly. Proceedings of the SIGCHI Conference on Human Factors in Computing Systems, Fort Lauderdale, FL, 73-80. https://doi.org/10.1145/642611.642626

Tsai, P. S., Tsai, C. C., \& Hwang, G. H. (2010). Elementary school students' attitudes and self-efficacy of using PDAs in a ubiquitous learning context. Australasian Journal of Educational Technology, 26(3), 297-308. Retrieved from https://ajet.org.au/index.php/AJET/article/view/1076/333

Vandenbroeck, M., Verschelden, G., \& Boonaert, T. (2008). E-learning in a low-status female profession: The role of motivation, anxiety and social support in the learning divide. Journal of Computer Assisted Learning, 24(3), 181-190. https://doi.org/10.1111/j.1365-2729.2007.00252.x

Vate-U-Lan, P. (2012). An augmented reality 3D pop-up book: The development of a multimedia project for English language teaching. Proceedings 2012 IEEE International Conference on Multimedia and Expo, Melbourne, Australia, 890-895. https://doi.org/10.1109/ICME.2012.79

Wang, J. L., Jackson, L. A., Wang, H. Z., \& Gaskin, J. (2015). Predicting Social Networking Site (SNS) use: Personality, attitudes, motivation and Internet self-efficacy. Personality and Individual Differences, 80, 119-124. https://doi.org/10.1016/j.paid.2015.02.016

Wojciechowski, R., \& Cellary, W. (2013). Evaluation of learners' attitude toward learning in ARIES augmented reality environments. Computers \& Education, 68, 570-585. https://doi.org/10.1016/j.compedu.2013.02.014

Corresponding author: Kun-Hung Cheng, kuhu@mail.nctu.edu.tw

Australasian Journal of Educational Technology (C) 2017.

Please cite as: Cheng, K. H. (2017). Reading an augmented reality book: An exploration of learners' cognitive load, motivation, and attitudes. Australasian Journal of Educational Technology, 33(4), 5369. https://doi.org/10.14742/ajet.2820 


\section{Appendix A \\ Items for cognitive load survey}

A1. Mental effort

1. When I read the AR book, I have to understand the content of the learning material by investing more mental effort.

2. When I read the AR book, I have to invest much mental effort to figure out the augmented information.

A2. Mental load

1. When I read the AR book, the way in which the learning material is explained gives me a lot of pressure.

2. When I read the AR book, I cannot concentrate on the learning material. 


\section{Appendix B \\ Items for motivation survey}

B1. Attention

1. The AR book can draw my attention.

2. I am curious about the information provided by the AR book.

3. Reading paper book with the aid of AR technology is novel to me.

4. I pay my attention to read the AR book continuingly.

B2. Confidence

1. I belief that the AR book can help users to learn.

2. I belief that reading with the aid of AR technology can be helpful for understanding the content of the book more.

3. I am confident in the benefits of AR technology on reading.

B3. Satisfaction

1. I like to read the AR book.

2. I I am dissatisfied with the experiences of the AR book reading.*

3. I feel happy when I read the AR book.

* Scored in a reverse way 


\section{Appendix C \\ Items for attitudes survey}

C1. Perceived control

1. I think the AR book system is easy to use.

2. I can learn to use the AR book system in a short time.

3. The AR book system is difficult to use for me.*

4. I need an experienced person nearby when I read the AR book.*

C2. Perceived usefulness

1. I think the AR book is helpful for my learning.

2. The AR book can help me to understand the content more clearly.

3. Reading the AR book is helpful for generating more ideas.

4. The AR book reading is an alternative learning method.

C3. Behaviour of learning

1. After reading the AR book, I want to understand Hakka culture in more depth.

2. After reading the AR book, I have more interests in Hakka culture.

3. I hope to read more information regarding the topic of the AR book.

4. Reading the AR book did not foster my interests in Hakka culture.*

5. The issues regarding Hakka culture were not attractive for me even I have read the AR book.*

C4. Behaviour of AR learning

1. I hope to have more opportunities to learn with the aid of AR technology.

2. I am inclined to seek AR books with other topics in the future.

3. I expect that there are more applications of AR technology on learning.

4. I have more intention to read book by AR technology.

* Scored in a reverse way 